\title{
Conical epidermal cells cause velvety colouration and enhanced patterning in Mandevilla flowers
}

\author{
Doekele G. Stavenga, (iD a Marten Staal ${ }^{b}$ and Casper J. van der Kooi iD b
}

Received 11th May 2020, Accepted 1st June 2020

DOI: $10.1039 /$ dOfd00055h

The majority of angiosperms have flowers with conical epidermal cells, which are assumed to have various functions, such as enhancing the visual signal to pollinators, but detailed optical studies on how conical epidermal cells determine the flower's visual appearance are scarce. Here we report that conical epidermal cells of Mandevilla sanderi flowers effectively reduce surface gloss and create a velvety appearance. Owing to the reduction in surface gloss, the flower further makes more efficient use of floral pigments and light scattering structures inside the flower. The interior backscattering yields a cosine angular dependence of reflected light, meaning that the flowers approximate near-perfect (Lambertian) diffusers, creating a visual signal that is visible across a wide angular space. Together with the large flowers and the tilted corolla tips, this generates a distinct visual pattern, which may enhance the visibility to pollinators.

\section{Introduction}

The vast majority of angiosperms have flowers with conical epidermal cells, which may have different roles in pollination. For example, conical epidermal cells may reduce petal wettability and/or provide grip or tactile cues to landing insect pollinators. ${ }^{1,2}$ Another hypothesis for the function of the cones is that they act as small lenses to enhance light capture by the pigments in the epidermal cells and increase colour contrast. ${ }^{3-6}$ However, conical epidermal cells generally vary in size and spacing and how this determines possible optical effects is unknown, particularly under natural conditions where the illumination varies. ${ }^{7}$

Here, we put forward a new function of conical epidermal cells, namely that the cones reduce surface gloss and so increase the flower's contrast. We have

${ }^{a}$ Surfaces and Thin Films, Zernike Institute for Advanced Materials, University of Groningen, NL-9747 AG Groningen, The Netherlands. E-mail: D.G.Stavenga@rug.nl

${ }^{b}$ Groningen Institute for Evolutionary Life Sciences, University of Groningen, NL-9747 AG Groningen, The Netherlands. E-mail: C.J.van.der.Kooi@rug.nl 
chosen Mandevilla sanderi (also known as Dipladenia) flowers to study the optical characteristics of conically-shaped epidermal cells because the flowers, when observed from various directions, display distinctly varied reflection patterns with a velvety appearance. This intriguing phenomenon presumably has a structural origin, which inspired us to further investigate the flowers' spatial colouration characteristics.

Mandevilla plants, also known as rocktrumpets, are popular garden plants due to their strikingly coloured, large flowers. The genus Mandevilla belongs to the family Apocynaceae, and its members differ in floral traits such as corolla shape, colour and size. ${ }^{8}$ Mandevilla species are pollinated by different guilds of pollinators, including bees, ${ }^{9}$ hummingbirds ${ }^{10}$ and hawkmoths. ${ }^{11,12}$ Notably, the Sundaville varieties of Mandevilla sanderi have large flowers with a brightly red, pink, yellow or white coloured five-lobed corolla. The 'Sundaville Red' variety has a deep-red colour due to strongly anthocyanin-pigmented epidermal cells. The cone shape of the flower's epidermal cells is similar in size and shape to those found in flowers of many species. ${ }^{\mathbf{4}, 13-15}$ Measurements of the flowers' reflectance spectra show that the conical shape of the adaxial epidermal cells effectively reduces gloss, especially when observed under large angles. As a consequence, tilted corolla tips become much darker than untilted lobe areas, and in this way contrasting, velvety flower patterns are created.

\section{Materials and methods}

\section{Plant material, photography, and anatomy}

Two 'Sundaville Red' Mandevilla sanderi plants were obtained from a commercial supplier. The anatomical, reflection and pigmentation characteristics of the plants were very similar. Macro-photographs of the flowers were obtained with a Canon DC7 digital camera. To visualize the location of the red pigment, flower pieces were embedded in a $6 \%$ agarose solution at a temperature of approximately $55{ }^{\circ} \mathrm{C}$, i.e. close to the temperature of agarose solidification. Micrographs of transverse sections were subsequently obtained with a Zeiss Universal microscope (Zeiss, Oberkochen, Germany), equipped with an Epiplan 16/0.35 objective and a DCM50 camera (Mueller-Optronic, Erfurt, Germany). The microscope was also used for photographing the reflection and transmission of flower lobes.

\section{Spectrophotometry}

Reflectance spectra were measured as a function of angle of light incidence and reflection in a goniometric setup with two rotatable optical fibers. One fiber delivered light from a xenon lamp to the object, and the other fiber collected the reflected light and guided it to an AvaSpec-2048 spectrometer (Avantes, Apeldoorn, The Netherlands). The angular resolution of the setup has a Gaussian shape with half-width $\sim 5^{\circ} .{ }^{16}$ All measured spectra were divided by the spectrum obtained from a white diffuse reflectance standard (WS-2, Avantes), which was illuminated normally while the detector was also positioned in the normal direction. The measurements were mainly performed with unpolarized light on five lobes, yielding very similar results. 


\section{Results}

\section{Flower structure and the shape of epidermal cells}

The Red morph of the Mandevilla flower has a five-lobed corolla, coloured deep-red (Fig. 1). While the adaxial side of the lobes is matt (Fig. 1a and b), with varying brightness across the lobes' plane, the abaxial side is glossy (Fig. 1c). Cross-sections of the Red morph's lobes revealed that the colour is due to pigment concentrated in both the adaxial (upper) and abaxial (lower) epidermis (Fig. 1d). The adaxial epidermal cells have a distinctly conical papillate shape, but the abaxial epidermal cells are only slightly convex. The mesophyll in between the epidermises is interspersed with large air holes (Fig. 1d).

Due to the different shapes of the epidermal cells, the adaxial and abaxial surfaces have a different appearance. When observed with an epi-illumination light microscope, the conical cells of the adaxial epidermis appear to be arranged in a rather orderly manner in an approximately hexagonal lattice. Focusing at the level of the cone tips reveals distinct surface reflections (Fig. 2a), and at a deeper level the conical cell borders emerge (Fig. 2b). When changing the epi-illumination to transmitted light, bright dots occur at a level about halfway in between the cell tips and borders, clearly marking the level of the focal points of the conical cells (Fig. 2c). Focusing at the level of the cell borders, the transmitted light shows bright border lines surrounding dark-red circles (Fig. 2d), indicating that the red pigment is homogeneously distributed in the cone cells, in agreement with the anatomy of Fig. 1d.
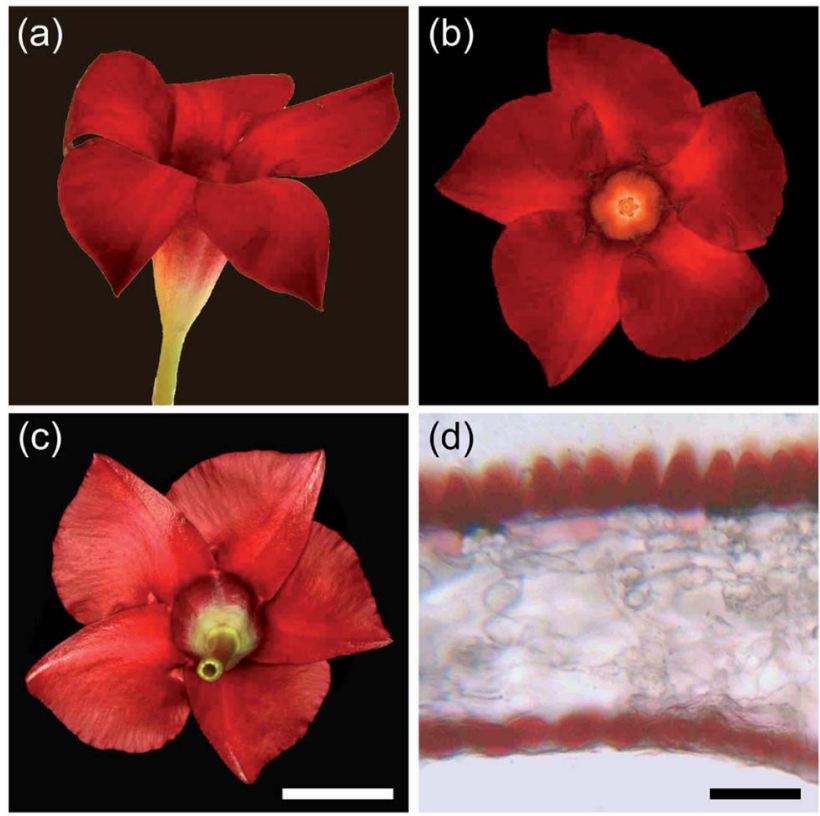

Fig. 1 Mandevilla 'Sundaville Red' flower. (a) Lateral view. (b) Upper side view. (c) Underside view. (d) Lobe section embedded in agarose. Scale bars: (a-c), 2 cm; (d), $50 \mu \mathrm{m}$. 

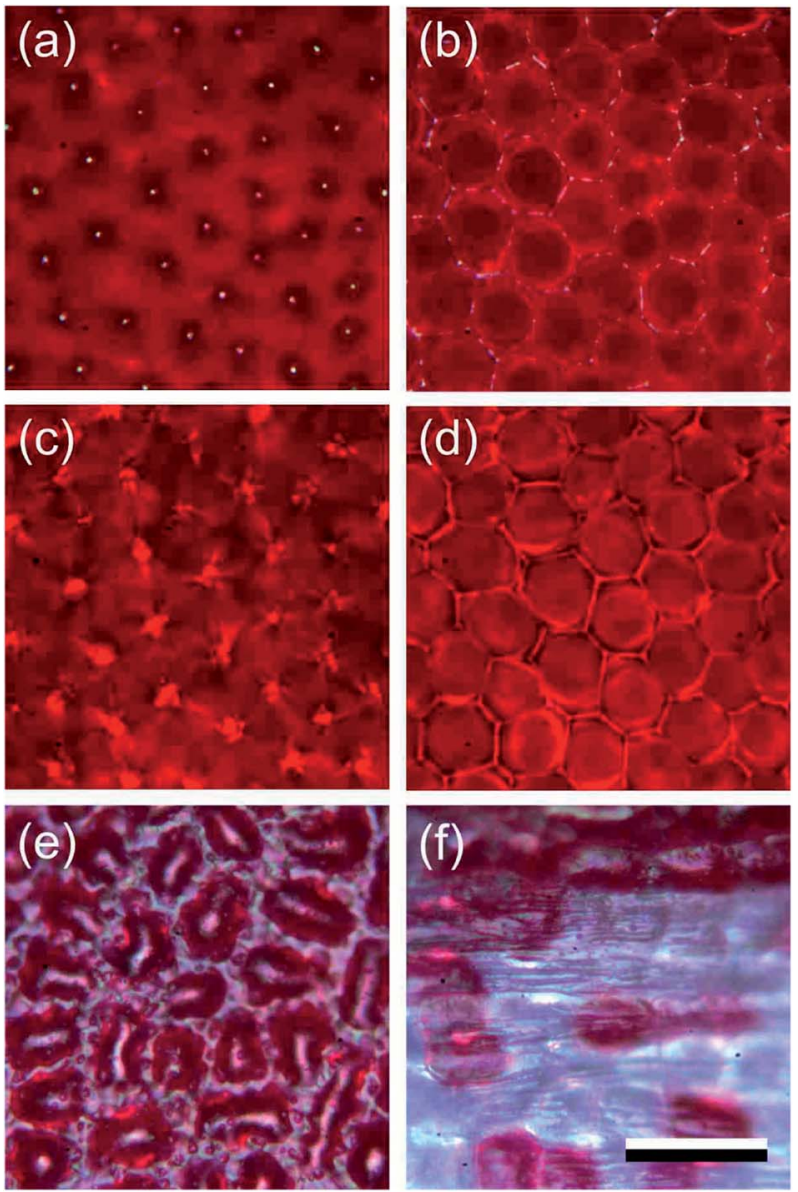

Fig. 2 Close-up views of the lobe epidermis of the Red morph. (a) Focus at the adaxial cone tips. (b) Level of cone cell borders. (c) Level of focal points of the cone cells. (d) Level of cone cell borders. (e) Heavily pigmented area of lower epidermis. (f) Sparsely pigmented area proximally in the lower epidermis in the transition zone of lobe and tube. (a-d) Adaxis; (e and f) abaxis; ( $a, b$, e and f) epi-illumination; (c and d) transmitted light. Scale bar: ( $a-f$ ), $50 \mu \mathrm{m}$.

Epi-illumination of the abaxial side shows the more or less random arrangement of the red-pigmented epidermal cells (Fig. 2e). The picture is glossy due to the fairly smooth surface of the slightly convex epidermal cells (Fig. 1d). In the more proximal corolla area, in the transition zone of the lobe to the tube, the pigmentation of the abaxial epidermal cells vanishes stochastically (Fig. 2f), so that a greenish to colourless tube and peduncle remain (Fig. 1a and c).

\section{Reflectance spectra of the different flower areas}

To better understand the optical mechanisms causing the different appearances of the matt adaxial and glossy abaxial lobe sides, we studied the spectral characteristics of the corolla lobes using angle dependent reflectance measurements. We applied spectrophotometry to both the adaxial and abaxial sides of the corolla 
lobes using a goniometric setup with two rotatable fibers, one delivering the illumination and the other collecting the reflected light, while systematically varying the illumination or detection angle.

We firstly applied normal illumination and measured the reflectance at various reflection angles (Fig. 3a; see inset). For all angles of reflection, the reflectance of the lobe's adaxial side is very low throughout the main visible wavelength range. In the longer wavelength range, the reflectance is high, but it decreases monotonically with an increasing angle of reflection (Fig. 3a). The reflectance of the abaxial side, when measured with the same procedure, is much higher, especially for normally incident light (Fig. 3b). To assess the angle dependence of the reflectance of both flower sides, we evaluated the reflectance at 550 and $750 \mathrm{~nm}$ separately (Fig. 3c and d). Clearly, the adaxial reflectance at $550 \mathrm{~nm}\left(R_{550}\right)$ is negligible for all reflection angles (Fig. 3a and c), but the abaxial $R_{550}$ is considerable for angles up to $\sim 30^{\circ}$ (Fig. $3 \mathrm{~d}$, blue curve); the latter is due to the surface gloss (Fig. $3 \mathrm{~b}$ and d). Given that the floral pigment absorbs strongly between 300 and $600 \mathrm{~nm}$, the $R_{550}$ is completely due to surface reflections. Assuming that this surface gloss is the same for all wavelengths, subtracting $R_{550}$ from the reflectance at $750 \mathrm{~nm}\left(R_{750}\right)$ yields the backscattering from the lobe interior, $R_{\mathrm{i}}=R_{750}-R_{550}$, which approximates a cosine function for both the adaxial and abaxial sides (Fig. $3 \mathrm{c}$ and d). Such a cosine-angular dependence of the reflectance is characteristic of a Lambertian, matte and diffusely reflecting surface, indicating that the flower interior approximates an ideal reflecting diffuser. Yet, for a perfect Lambertian diffuser the amplitude at normal
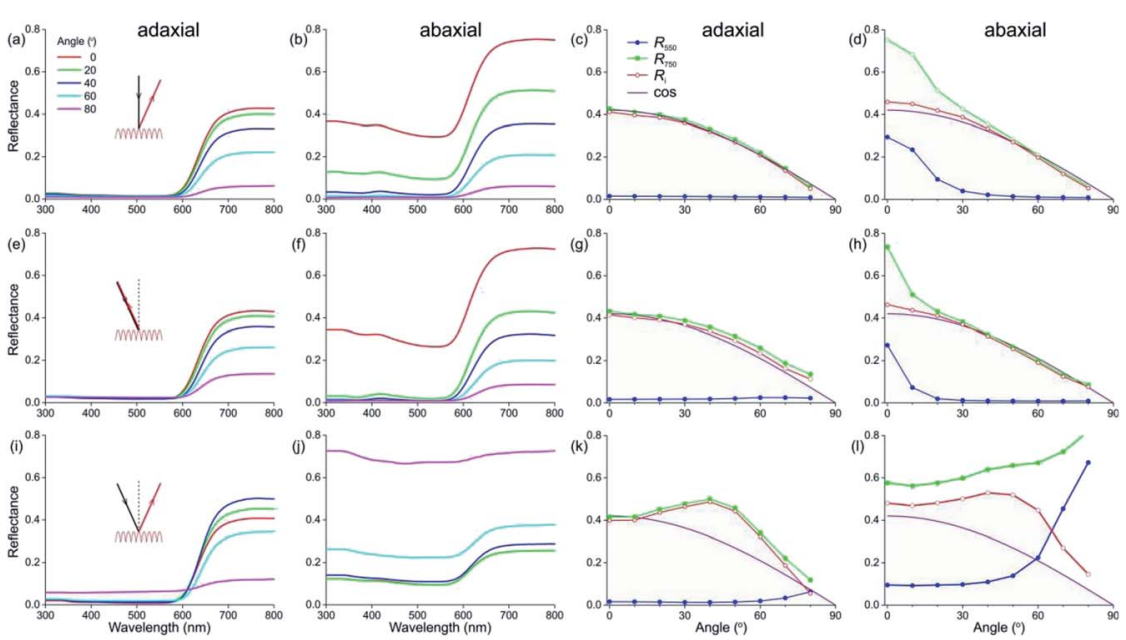

Fig. 3 Angle-dependent reflectance of the adaxial and abaxial sides of a Red morph corolla lobe. (a-d) Illumination (inset, black) normal and stable; detector angle (inset, red) varying. (e-h) Illumination and detector angle identical and varying. (i-l) Illumination and detector at different angles symmetrical with respect to the normal. ( $a, b, e, f, i$ and j) Reflectance spectra measured at angles $0^{\circ}, 20^{\circ}, 40^{\circ}, 60^{\circ}$, and $80^{\circ}$ with respect to the normal. (c, d, g, h, k and l) Reflectance values at 550 and $750 \mathrm{~nm}\left(R_{550}\right.$ and $\left.R_{750}\right)$ and their difference $\left(R_{\mathrm{i}}=R_{750}-R_{550}\right)$ as a function of the detector angle, compared with a cosine function (cos). (a, c, e, g, i and k) Measurements at adaxial side. (b, d, f, h, j and l) Measurements at abaxial side. 
illumination is 1 , whereas for the lobe interior it is 0.42 , which is due to the limited thickness of the lobe.

We subsequently varied the illumination angle and measured the light reflected into the same angle (Fig. 3e; see inset). The reflectance spectra measured for the adaxial and abaxial side were surprisingly similar to those of the previous case where the illumination was always normal. Indeed, processing the spectral data in the same way as above revealed that the reflectance difference $R_{\mathrm{i}}=R_{750}-R_{550}$ approximated the same cosine function as that of Fig. $3 \mathrm{c}$ and d (Fig. $3 \mathrm{~g}$ and h). Only the angular spread of $R_{550}$ was now slightly narrower (comparing Fig. $3 \mathrm{~h}$ with $3 \mathrm{~d}$ ).

In a third approach, we positioned the illumination and detector at opposite, mirror angles (Fig. 3i-l). The reflectance of the adaxial side measured this way was again in the main part of the visible wavelength range minimal except for extremely oblique angles; in other words, $R_{550}$ was minor except for angles $>70^{\circ}$ (Fig. 3k). However, the angle-dependence of the reflectance component due to backscattering by the flower's interior, $R_{\mathrm{i}}$, deviated from the cosine function, showing a slightly enhanced reflectance for angles of incidence and reflection around $40^{\circ}$ (Fig. $3 \mathrm{k}$ ).

The abaxial reflectance behaved very differently. The considerable reflectance throughout the whole wavelength range rapidly increased with an increasing angle of light incidence and reflection (Fig. 3j). When subtracting the measured abaxial $R_{550}$ from $R_{750}$, the resulting angle dependence of the interior reflectance was highly similar to the corresponding data deduced for the adaxial side (red curves in Fig. 3k and i), meaning that the arrangement of interior structures is random. However, for low values of the angle of incidence $R_{550}$ was approximately constant, but it rapidly rose for angles $>45^{\circ}$, yielding reflectance values $>1$ for angles $>60^{\circ}$. These unrealistically high values were obtained because the spectrum of a normally-illuminated ideal diffuser was used as a reference. The assumed criterion of a diffuser holds for the adaxial surface (Fig. 3c), but for the abaxial surface it also holds only when the angles of light incidence and reflection widely differ, i.e. $>30^{\circ}$ (e.g. Fig. $3 \mathrm{~d}$ and h). Therefore, when measuring the reflectance of the abaxial flower surface in the mirror angle, the detector will capture a large fraction of the surface reflections in addition to the (comparatively low) backscattering of the lobe interior. We estimated that the specularity of the abaxial side causes an overestimate of the reflectance by a factor of $\sim 3$, and therefore in Fig. $3 \mathrm{j}$ we present the measured spectra divided by 3 . Fig. 3 f contains the associated values of $R_{550}$ (as well as the values of $R_{750}$, now being the sum of $R_{\mathrm{i}}$ and $R_{550}$ ).

To ascertain that the reflectances of the abaxial side measured in the shortwavelength range were indeed virtually totally due to the surface reflections, as a control we also performed the same series of measurements using polarized light, by fitting the detector fiber with a linear analyzer. The $R_{550}$ data for TE- and TM-polarized light (that is, polarized perpendicular and parallel to the plane of light incidence, respectively) were as expected for a reflecting dielectric medium, with the TE-reflectance rising monotonically and the TM-reflectance approaching zero for an angle of light incidence $\sim 60^{\circ}$. As expected for a diffuser, the interior reflectance $R_{\mathrm{i}}$ was virtually independent of the polarization (not shown).

\section{Discussion}

Our analysis of the angle-dependent reflections of Mandevilla flowers demonstrates that two clearly distinguishable mechanisms contribute to the flower 
reflectance, i.e. firstly the reflecting surface and secondly the flower interior that backscatters incident light. The conclusion that both the surface and interior of flowers contribute to the visual signal has been shown before, ${ }^{4,717-19}$ but the relative contributions of the surface and interior and how they depend on the angles of illumination and observation has remained virtually unstudied.

For the adaxial flower side we found that the surface reflections are minimal in the wavelength range up to $\sim 600 \mathrm{~nm}$ for all angles of light incidence and reflection. Therefore, the considerable reflectance measured in the longwavelength range must be due to scattering inhomogeneities in the flower interior. The interior backscattering results in a cosine angular dependence of the diffused light, i.e. highly similar to the case of a Lambertian surface. For the abaxial side, the approximately smooth surface creates reflections that are far from negligible, and even creates a slightly metallic lustre, which can also be found in other species. ${ }^{20,21}$ When illuminated with a narrow-aperture light source, the abaxial surface reflections show a minor angular spread (half-width 10-15 ${ }^{\circ}$ ), owing to the slightly convex surfaces of the abaxial epidermal cells.

Whereas the reflections of the adaxial and abaxial flower surfaces are very different, the light backscattered by the interior as seen from the adaxial and abaxial sides is remarkably similar (Fig. 3d, h and l). Furthermore, for both sides, when the angles of light incidence and reflection are equal but opposite, the angular dependence of the interior reflectance modestly departs from that of an ideal diffuser. Presumably the directional component of the reflectance is due to some planar arrangement of the lobe's interior structures, such as the stratification of interior cell layers.

The cosine angle dependence of the long-wavelength reflectance has interesting consequences for flowers with tilted tips, as is the case for Mandevilla flowers (Fig. 1). The corolla features a contrasting pattern, in spite of the uniform red pigmentation across the corolla lobes. In principle this could also be the case when observing the abaxial side of the flower lobe, but the gloss of the surface reflections drowns the interior reflections. Furthermore, as the gloss is independent of wavelength, it will severely diminish the colour contrast, which is a critical aspect for detection by insect pollinators. ${ }^{7}$

The epidermal cone cells thus have a crucial function in reducing gloss and enhancing colour contrast via two different optical processes. A long-standing hypothesis is that enhanced colouration is achieved by light focusing onto the pigment. $^{3,4,22}$ A similar colour-enhancing function has been attributed to the ridges of the elongated petal epidermal cells of the California poppy (Eschscholzia californica). ${ }^{22}$ We note that the cones may indeed function as lenses (Fig. 2c), but the focusing will strongly depend on the direction of the incident light, so that with wide-angled, natural illumination there is no distinct focusing. Thus, rather than having a focusing function, the actual optical function of the cone-shaped adaxial epidermal cells is to effectively annihilate the gloss, which undermines the colour contrast that is pivotal in the visual detection of flowers by pollinators. ${ }^{7}$

In addition to reducing surface gloss, a decreased surface reflectance means more light will enter the flower and reach the floral pigments. This will have severe effects, especially for incident light at oblique angles. A larger fraction of incident light entering the flower interior results in an increased backscattering by the diffusing structural components. Further, light that enters the flower will be filtered by pigments present in the epidermal cell layer (Fig. 1d). When the 
light is subsequently backscattered by the interior structures it traverses the pigment layer a second time, ${ }^{18}$ meaning that the light reflected by the flower interior is modulated even more and exhibits a high colour contrast against the surrounding vegetation. In summary, instead of having a focusing function, conical epidermal cells enhance colour contrast by both decreasing surface gloss and increasing long-wavelength reflectance.

A contrasting case is that of buttercups, which instead of decreasing surface reflectance increase the adaxial epidermal reflection. Their adaxial epidermis is a carotenoid-filled thin film in air, which causes a high yellow reflectance. ${ }^{23,24}$ The petals of buttercups together form a paraboloid mirror, and as the flowers are heliotropic, they keep sunlight focused at the reproductive organs, presumably to increase flower temperature. ${ }^{24}$ This mechanism will not work in flowers with a spread-out corolla, for which a rough surface is then advantageous.

Gloss reduction by surface roughening is also a widespread trait in the animal kingdom for reducing specularity and/or enhancing transmittance. ${ }^{25-28}$ Additional or alternative roles for rough flower surfaces could be, for example, antiwettability and self-cleaning. ${ }^{15,29,30}$ Furthermore, the conical epidermal cells of flowers may enhance grip for landing insect pollinators, ${ }^{2,19}$ but this is not underscored by the recent finding that flowers pollinated by landing insects (bees and flies) do not have more cone-shaped surfaces than flowers pollinated by animals that do not land on flower surfaces (birds and hawkmoths) or via selfpollination. ${ }^{\mathbf{1 4}}$

A main function of the conically-shaped adaxial cells of the adaxial epidermis is to create a visual signal that is widely visible and, in the case of large, pleated and deeply-pigmented flowers, to create contrasting patterning in the lobe. The increase of within-flower colour contrast and the scattering of light into a wide angular space will increase the flower's visibility to pollinators. How conical cells contribute to colour formation in species with other pigmentation and how this enhances flower salience in natural conditions provides an intriguing avenue for future research.

\section{Conflicts of interest}

There are no conflicts to declare.

\section{Acknowledgements}

We thank Dr Bodo Wilts for providing constructive comments and Hein Leertouwer for technical assistance. This study was financially supported by the AFOSR/EOARD (grant FA9550-15-1-0068, to DGS) and NWO (Veni grant 016.Veni.181.025, to CJvdK).

\section{References}

1 P. G. Kevan and M. A. Lane, Proc. Natl. Acad. Sci. U. S. A., 1985, 82, 4750-4752.

2 H. M. Whitney, K. V. Bennett, M. Dorling, L. Sandbach, D. Prince, L. Chittka and B. J. Glover, Ann. Bot., 2011, 108, 609-616.

3 F. Exner and S. Exner, Sitzungsber. Kais. Akad. Wiss. Wien, Math.-Nat. Kl. I, 1910, 119, 191-245. 
4 Q. Kay, H. Daoud and C. Stirton, Bot. J. Linn. Soc., 1981, 83, 57-83.

5 R. A. Bone, D. W. Lee and J. Norman, Appl. Opt., 1985, 24, 1408-1412.

6 T. C. Vogelmann, J. F. Bornman and D. J. Yates, Physiol. Plant., 1996, 98, 43-56.

7 C. J. van der Kooi, A. G. Dyer, P. G. Kevan and K. Lunau, Ann. Bot., 2019, 123, 263-276.

8 L. De Araújo, Z. Quirino and I. Machado, Plant Biol., 2014, 16, 947-955.

9 C. Löhne, I. C. Machado, S. Porembski, C. Erbar and P. Leins, Bot. Jahrb. Syst. Pflanzengesch. Pflanzengeogr., 2004, 125, 229-243.

10 F. G. Stiles and C. E. Freeman, Biotropica, 1993, 25, 191-205.

11 M. Moré, A. N. Sérsic and A. A. Cocucci, Ann. Mo. Bot. Gard., 2007, 94, 485-505.

12 A. Rubini Pisano, M. Moré, M. A. Cisternas, R. A. Raguso and S. Benitez-Vieyra, Plant Biol., 2019, 21, 206-215.

13 P. Bräuer, C. Neinhuis and D. Voigt, Arthropod Plant Interact., 2017, 11, 171192.

14 M. Kraaij and C. J. van der Kooi, Plant Biol., 2020, 22, 177-183.

15 A. Watanabe-Taneda and H. Taneda, Flora, 2019, 257, 151417.

16 D. G. Stavenga, B. D. Wilts, H. L. Leertouwer and T. Hariyama, Philos. Trans. $R$. Soc., B, 2011, 366, 709-723.

17 S. Vignolini, M. P. Davey, R. M. Bateman, P. J. Rudall, E. Moyroud, J. Tratt, S. Malmgren, U. Steiner and B. J. Glover, New Phytol., 2012, 196, 1038-1047.

18 D. G. Stavenga and C. J. van der Kooi, Planta, 2016, 243, 171-181.

19 S. Papiorek, R. R. Junker and K. Lunau, PLoS One, 2014, 9, e112013.

20 Y. Zhang, T. Hayashi, M. Hosokawa, S. Yazawa and Y. Li, Sci. Hortic., 2009, 121, 213-217.

21 Y. Zhang, T. Sun, L. Xie, T. Hayashi, S. Kawabata and Y. Li, J. Plant Res., 2015, 128, 623-632.

22 B. D. Wilts, P. J. Rudall, E. Moyroud, T. Gregory, Y. Ogawa, S. Vignolini, U. Steiner and B. J. Glover, New Phytol., 2018, 219, 1124-1133.

23 S. Vignolini, M. M. Thomas, M. Kolle, T. Wenzel, A. Rowland, P. J. Rudall, J. J. Baumberg, B. J. Glover and U. Steiner, J. R. Soc., Interface, 2012, 9, 12951301.

24 C. J. van der Kooi, J. T. M. Elzenga, J. Dijksterhuis and D. G. Stavenga, J. R. Soc., Interface, 2017, 14, 20160933.

25 I. R. Hooper, P. Vukusic and R. Wootton, Opt. Express, 2006, 14, 4891-4897.

26 M. Spinner, A. Kovalev, S. N. Gorb and G. Westhoff, Sci. Rep., 2013, 3, 1846.

27 D. L. Maurer, T. Kohl and M. J. Gebhardt, Arthropod Struct. Dev., 2017, 46, 147155.

28 J. Riedel, M. J. Vucko, S. P. Blomberg, S. K. Robson and L. Schwarzkopf, J. Anat., 2019, 234, 853-874.

29 H. Taneda, A. Watanabe-Taneda, R. Chhetry and H. Ikeda, Ann. Bot., 2015, 115, 923-937.

30 W. Barthlott and C. Neinhuis, Planta, 1997, 202, 1-8. 